\section{New clue to Ras lipid glue}

\section{Douglas R. Lowy and Berthe M. Willumsen}

Normal Ras proteins encoded by the ras genes and oncogenic Ras proteins encoded by mutated ras genes (oncogenes) are synthesized as a precursor in the cell cytoplasm and then modified and translocated to the cytoplasmic face of the cell's outer membrane ${ }^{1}$. Localization to the membrane is critical for efficient Ras function, as the biological activity of those mutant Ras proteins that remain in the cytosol is severely impaired ${ }^{2}$. Until very recently, most investigators believed that it was the attachment of palmitate near the carboxy-terminus of Ras precursors that mediated their movement to the plasma membrane ${ }^{3}$. Now, evidence from three laboratories ${ }^{46}$, each using a distinct approach and making a unique contribution, indicates that the lipid invariably added to mature Ras proteins is a farnesyl isoprenoid, and that palmitate is only an optional extra. The farnesyl moiety common to all mature Ras proteins is derived from farnesol, which is a branchedchain, polyunsaturated hydrocarbon alcohol intermediate of sterol biosynthesis. As farnesol is derived from mevalonate, pharmacologic agents which interfere with 3-hydroxy-3-methylglutaryl coenzyme A (HMG CoA) reductase (the enzyme that catalyses mevalonate synthesis), or other steps before farnesol synthesis, may be able to inhibit membrane localization of Ras protein, thus providing a potential approach to interfere with Ras-protein function.

The critical clues leading to these findings came from the recognition of the similarities between the post-translational modifications of fungal mating factors, such as yeast a-factor ${ }^{3,7}$ and those of Ras. Indeed, a yeast mutant defective in posttranslational modification of mating factor seems to cause a similar defect in yeast $\operatorname{Ras}^{5.8}$. The last four amino acids of both a-factor and Ras (see figure), when first translated, can be described as Cys-A-A-X, where Cys is cysteine, $\mathbf{A}$ is an aliphatic amino acid (most often the hydrophobic amino acids leucine, isoleucine or valine), and $\mathrm{X}$ is any amino acid $^{3}$. The carboxy-terminus of the mating factor has been shown to undergo several post-translational changes, including proteolytic cleavage of the $\mathrm{A}-\mathrm{A}-\mathrm{X}$, carboxymethylation of the (now carboxyterminal) cysteine and its farnesylation via thioether linkage to its sulphur atom ${ }^{7}$. Virtually identical modifications of Ras have now been shown to occur ${ }^{4-6,9,10}$. Furthermore, a Cys-A-A-X box, when placed at the carboxy-terminus of a protein that does not normally have one, is sufficient to direct its binding to an isoprenoid (presumably a farnesyl moiety) ${ }^{4}$.
Palmitate, which was formerly believed to be linked to the cysteine residue in the Cys-A-A-X box, has now been shown to be linked instead to cysteine residues located in the first few amino acids upstream from the box. Moreover, the palymitoylation somehow depends upon post-translational modification of the box ${ }^{2,4}$. Rather than representing an obligatory step in Ras-protein function, palmitoylation can now be viewed as a modification that may modulate the biological activity of a given Ras protein. Supporting this hypothesis, palmitoylated Ras proteins have been found to be more firmly anchored in the membrane than non-palmitoylated versions and to be more active in stringent bioassays ${ }^{4}$. Most eukaryotes, including mammals, contain multiple ras genes that encode proteins with considerable sequence divergence in the 20 amino acids immediately upstream from their Cys-A-A-X box'. This sequence divergence is associated with variation in the precise location and number of cysteine residues that can be Palmitoylated (see figure). As the biological activity of a Ras protein seems to vary with the amount of palmitate that is added, each Ras protein may have its own particular level of activity, presumably to allow distinct functional roles to be better served than they could be by a

\title{
Giant sunspot clusters mark solar maximum
}

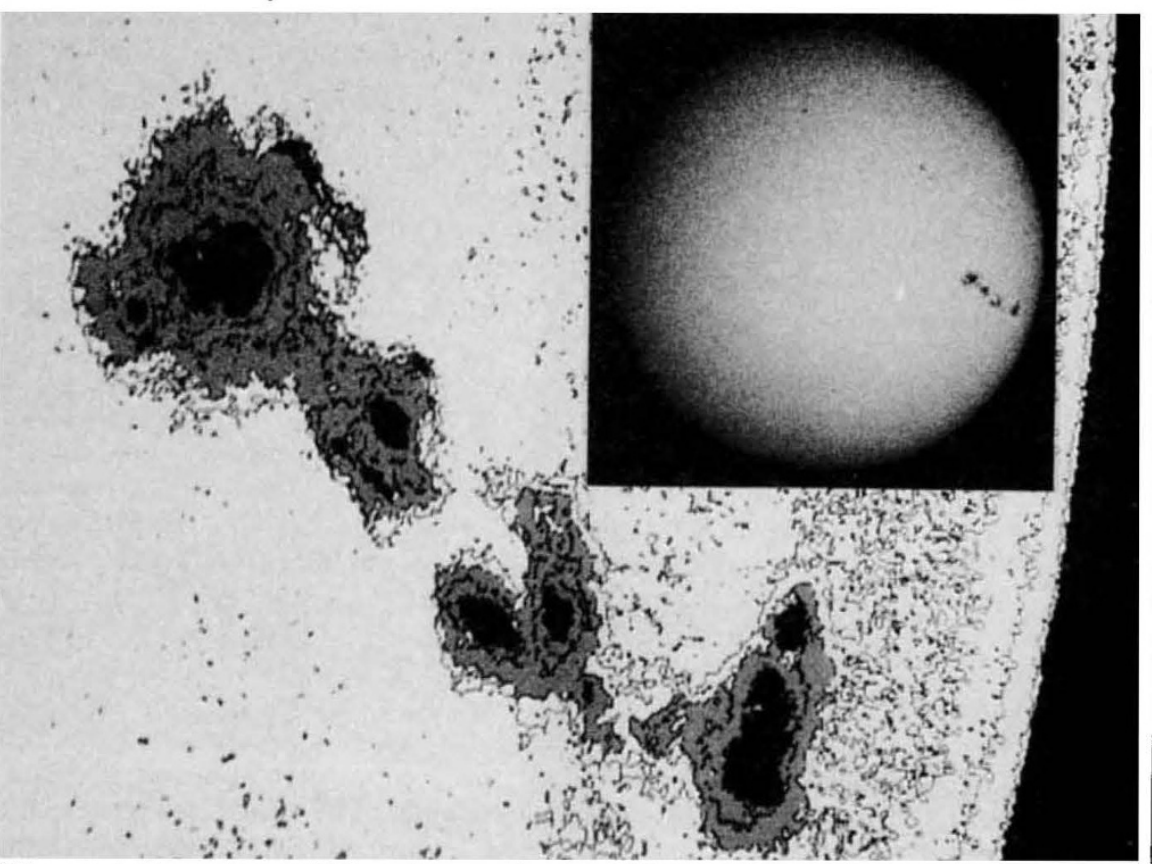

WITH only 3 months until the solar maximum, violent activity — flares and sunspots at the Sun's surface is increasing. This massive cluster of sunspots, photographed recently by N.W. Scott, traversed the Sun's face in 10-12 days. (Note: binoculars and telescopes should not be used for direct viewing of the Sun.) 